\title{
Bunting Identity and Mazur Identity for Non-Linear Elliptic Systems Including the Black Hole Equilibrium Problem
}

\author{
B. Carter
}

Groupe d'Astrophysique Relativiste, C.N.R.S., Observatoire de Paris, F-92125 Meudon, France

\begin{abstract}
Recent work by G. Bunting and by P. O. Mazur has developed new techniques for proving uniqueness theorems for extensive classes of non-linear elliptic boundary value problems including that of the equilibrium state of an electromagnetically charged black hole. These methods are described and compared. It is shown that the rather general class of harmonic mappings that can be dealt with by the Bunting method (which needs no internal symmetry group) can be regarded as a generalisation of the particular (totally symetric) class of non-linear $\sigma$-models that can be dealt with by the Mazur method.
\end{abstract}

\section{Introduction and Background}

The purpose of this work is to give a coherent description of the methods of proving uniqueness theorems for extensive classes of non-linear elliptic boundary value problems that have been developed recently by the independent work of Bunting [1,2] and of Mazur [3]. It is shown that the particular class of non-linear $\sigma$-models (where the field lies in the quotient space of a non-compact Lie group by a maximal compact subgroup) to which the Mazur method applies can be considered as a special case of the more general class of harmonic (hypergeodesic) mappings (where the image space has negative curvature) to which the Bunting method applies.

The particular application that provided the original motivation for the development of these methods was a two-dimensional boundary problem for a well behaved but non-linearly self coupled elliptic system that was posed by the present author $[4,5]$ (see Appendix A) as the crux of a programme whose objective is to obtain a complete classification of all isolated black hole equilibrium states subject to the classical Einstein and Einstein-Maxwell field equations. The well known conclusion that for practical astrophysical purposes any isolated (asymptotically flat) pure vacuum black hole equilibrium state can be described by (a certain subclass of) the 2-parameter Kerr family of Einstein-solutions was effectively established by 1975 along lines originally sketched out by the present 
author [6] in 1971 and subsequently completed by the work of Hawking [7, 8], the present author [4], and Robinson [9]. However while this body of work was adequate for the purpose of justifying the exclusive use of the Kerr solutions for describing isolated black holes in realistic astrophysical applications, it nevertheless left over a considerable number of loose ends involving technical questions of rigour and generality. Although it is difficult to imagine a realistic astrophysical situation in which the charge on an isolated black hole could become or remain large enough to have any significant effect on the geometry, the loose end that received the most widespread attention, in view of its obvious academic interest, was that of generalizing the results to cover solutions of the electromagnetic Einstein-Maxwell equations as well as the pure vacuum Einstein equations. Despite the early progress achieved by Robinson [10] (in 1974) the demonstration of the sufficiency of (a subclass of) the 3-parameter Kerr-Newman family of Einstein-Maxwell solutions for describing the corresponding charged states remained until very recently on an only slightly more rigorous footing than had been achieved for the pure vacuum case in 1971.

Before describing the method by which Bunting [1,2] and Mazur [3] have at last obtained a rigourous proof of the uniqueness of the Kerr-Newman solutions of the basic two-dimensional elliptic boundary value problem (as specified in Appendix A), it is to be mentioned that this still leaves many other loose ends to be sorted out before the classical black hole equilibrium problem can be considered to have been completely tidied up. To start with, the reduction $[4,5]$ of the global geometric black hole equilibrium problem (involving an unknown metric) in 4-dimensions to a regular albeit non-linear elliptic boundary value problem (involving a known metric) in 2-dimensions, is still dependent on a number of technical assumptions justified by heuristic physical considerations rather than mathematical arguments. For example although the work of Hawking $[7,8]$, in conjunction with the earlier theorems of Israel $[11,12]$ (as completed by the work of Müller zum Hagen et al. [13], Robinson [14] and most recently Bunting [2] has provided an almost, though not quite, complete mathematical justification for the earlier heuristically based assumption [6] that the equilibrium solutions should be axisymmetric, and that individual black hole components should be topologically spherical, there is nevertheless (as far as the author knows) still no mathematical basis for the physically plausible assumption that only one such topologically spherical axisymmetric component can be present. Indeed it has been shown by Hartle and Hawking [15] that counterexamples exist as marginally stable limit cases, in which gravitational attraction is balanced by electrostatic repulsion, and the existence of more general counterexamples, in which a spin-spin contribution to the repulsion might also be present has still (as far as the author knows) not been excluded.

Even after the basic 2-dimensional boundary value formulation of the problem, as presented in Appendix A has been justified, subject to suitable caveats excluding unphysical limit cases, it remains that all the partial uniqueness results obtained by the author [6] and by Robinson [9, 10], as well as the complete demonstrations of Bunting [1,2] and Mazur [3], are entirely dependent on the regular elliptic character of the problem, which itself was established $[4,6]$, not as a mathematical necessity from the basic definition [6] of a black hole equilibrium 
state, but on the basis of the additional physically motivated assumption of causality, which guarantees the spacelike nature of the Killing vector generator of the axial symmetry action. It was for this reason that I chose to formulate the problem (as given in Appendix A) on the basis of the axisymmetry generator, instead of using the more traditional Ernst type formulation, as based on the Killing vector generator of the stationary symmetry, which would lead to a system of field equations that is irregular on an "ergosurface" except in the strictly static (non-rotating) case. I am strongly inclined to believe in the conjecture that no more general (i.e. other than Kerr-Newman) solutions of the boundary condition problem as formulated in Appendix A can exist, even if one drops the causality requirement (by allowing the variable $X$ to become locally negative), but a treatment of a different kind from that described below would be necessary to prove this.

\section{The Basic Mazur Identity}

Like the earlier less complete results $[6,9,10]$ the recent demonstrations by Bunting [1,2] and Mazur [3] of the uniqueness of solutions of the regular twodimensional formulation $[4,5]$ of the black equilibrium problem (as recapitulated in Appendix A) depend on the construction of an identity equating some positive definite function of the difference between two sets of values of the field variables or their gradients to the sum of another quantity that vanishes locally when the field equations are satisfied and of the divergence of another quantity whose surface integral over the boundary vanishes when the boundary conditions are satisfied. By an application of Green's theorem, one can use such an identity to establish that the positive definite function, and hence also its argument, must vanish throughout, thereby establishing the uniqueness of the solutions or at least of their gradients. The identity constructed by Mazur [3] includes as special cases the earlier more specialized identities constructed by Robinson $[9,10]$ (and hence, a fortiori, their common limit as originally constructed by the present author [6]) However whereas these predecessors were obtained by ad hoc, hit and miss, procedures, the new identity of Mazur is based on a very elegant construction that can easily be adapted to cover a wide class of related problems. The independently developed identity of Bunting [1,2] (to be described in Sect. 6) is based on an even more widely applicable construction but it is from some points of view less elegant.

The Mazur construction can be applied to any system in which the field variables (over a base space of, let us say, $n$ dimensions) can be expressed in terms of a positive definite (let us say $m \times m$ ) matrix field obeying field equations such that there is a conserved current matrix of the form

$$
\mathbf{J}^{\mu}=\varrho \phi^{-1} \cdot \boldsymbol{\phi}^{; \mu},
$$

where the dot denotes contraction of adjacent (covariant and contravariant) matrix indices while the semi-colon denotes covariant derivation with respect to a positive definite metric Riemannian metric $\mathbf{g}$ with components of the form $g_{\mu v}$ say, in terms of base space coordinates $x^{\mu}(\mu=1, \ldots, n)$, and the proportionality factor $\varrho$ is arbitrary positive scalar weighting function on the base space. The positivity 
requirement on the matrix $\phi$, with components $\phi_{\dot{a} b}$ say $(a, b=1, \ldots, n$, where a dot is used to distinguish conjugate vector space components in the complex case) necessarily entails the hermiticity requirement,

$$
\phi=\phi^{*},
$$

where $\phi^{*}$ is the transposed conjugate matrix, whose components are defined by

$$
\phi^{*}{ }_{a b}=\bar{\phi}_{b \dot{a}},
$$

where a bar indicates ordinary complex conjugation. With the usual convention that raised indices denote dual space components, the inverse matrix $\phi^{-1}$, will have components of the form $\phi^{-1 a b}$, while the current itself will have mixed (undotted) components of the form $J_{\mu b}^{a}$. In consequence of (2.2) the hermitian conjugate current, as defined by

$$
J^{* \mu}{ }_{\dot{a}}^{b}=\bar{J}_{a}^{\mu b}
$$

will be expressible in the form

$$
\mathbf{J}^{* \mu}=\boldsymbol{\phi} \cdot \mathbf{J}^{\mu} \cdot \boldsymbol{\phi}^{-1} .
$$

In terms of covariant differentiation, the conservation property (which of course applies also to the conjugate current) is expressible in the usual way as

$$
\mathbf{J}_{; \mu}^{\mu}=0 .
$$

The explicit demonstration that the particular example of the standard 2-dimensional formulation of the black hole boundary problem, as given in Appendix A, can in fact be expressed in this standard Mazur form will be postponed until later (Sect. 4 and Appendix B). We shall also postpone until the next section the discussion of the rather severe internal symmetry group requirements that are necessary for the consistency of the linearly independent components of the matrix conservation law (2.4) when the linearly independent components $\phi_{i b}$ are not all algebraically independent, but subject to non-linear restrictions as is the case for the particular application we have in mind. Although such group theoretical considerations are relevant for considerations of generality and applicability, their invocation is an unnecessary deviation in so far as the derivation of the basic Mazur identity is concerned. By provisionally leaving aside this aspect one can shorten the original derivation [3] so as to obtain a result of the required form in a very direct manner as follows.

Let us consider two different sets of values, $\phi_{[0]}$ and $\phi_{[1]}$ say, of the field variables, and (slightly modifying our previous notation convention [5]) let us use a bull's eye, ${ }^{\odot}$, to indicate the corresponding difference between the values of any functionals of these variables, so that in particular for the current defined by (2.1) we have

$$
\stackrel{\odot}{\mathbf{J}^{\mu}}=\mathbf{J}_{[1]}^{\mu}-\mathbf{J}_{[0]}^{\mu} .
$$

This current difference bears a very simple relationship to the gradient of a certain deviation matrix $\Delta$ defined by

$$
\Delta=\stackrel{\odot}{\phi} \cdot \phi_{[0]}^{-1}=\phi_{[1]} \cdot \phi_{[0]}^{-1}-1,
$$


where $\mathbf{1}$ (with components $\delta_{b}^{a}$ ) is the unit matrix, so that $\Delta$ vanishes if and only if (as one intends ultimately to prove) the two sets of field variables coincide. One obtains

$$
\varrho \Delta^{; \mu}=\phi_{[1]} \cdot \stackrel{\odot}{\mathbf{J}}^{\mu} \cdot \phi_{[0]}^{-1} .
$$

The Mazur construction can be effected by taking the divergence of this, which immediately gives

$$
\left(\varrho \Delta^{; \mu}\right)_{; \mu}=\phi_{[1]} \cdot\left\{{\stackrel{\odot}{\mathbf{J}^{\mu}} ; \mu}^{-1}\left(\varrho_{[1] \mu} \cdot \mathbf{J}_{[1]}^{\mu}-2 \mathbf{J}_{[1] \mu} \cdot \mathbf{J}_{[0]}^{\mu}+\mathbf{J}_{[0] \mu} \cdot \mathbf{J}_{[0]}^{\mu}\right\}\right) \cdot \phi_{[0]}^{-1} .
$$

One now invokes the consequence (2.3) of the hermicity property (2.2), which makes it possible to rewrite the terms above that are quadratic in the current as

$$
\phi_{[1]} \cdot\left(\mathbf{J}_{[1]}^{\mu} \cdot \stackrel{\odot}{\mathbf{J}}_{\mu}-\stackrel{\odot}{\mathbf{J}}_{\mu} \cdot \mathbf{J}_{[0]}^{\mu}\right) \cdot \phi_{[0]}^{-1}=\mathbf{J}_{[1]}^{* \mu} \cdot \phi_{[1]} \cdot \stackrel{\odot}{\mathbf{J}}_{\mu} \cdot \phi_{[0]}^{-1}-\phi_{[1]} \cdot \stackrel{\odot}{\mathbf{J}}_{\mu} \cdot \phi_{[0]}^{-1} \cdot \mathbf{J}_{[0]}^{\mu} \cdot
$$

Hence taking the trace, and using the abbreviated notation

$$
\Delta=\operatorname{tr}\{\Delta\}=\Delta_{\dot{a}}^{\dot{a}}
$$

one finally obtains the scalar identity

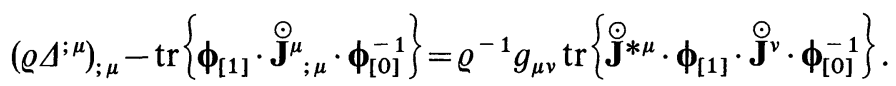

This has the required form, since the second term on the left-hand side drops out when one applies the field Eq. (2.4) which evidently implies

$$
{\stackrel{\odot}{\mathbf{J}^{\mu}}}_{; \mu}=0,
$$

while the term on the right-hand side will be a manifestly positive definite function of the current difference, i.e.

$$
{\stackrel{\odot}{\mathbf{J}^{\mu}}}^{\prime} 00 \varrho^{-1} g_{\mu \nu} \operatorname{tr}\left\{\stackrel{\odot}{\mathcal{J}^{* \mu}} \cdot \phi_{[1]} \cdot \stackrel{\odot}{\mathbf{J}}^{v} \cdot \boldsymbol{\phi}_{[0]}^{-1}\right\}>0
$$

as a consequence of the postulated positivity properties of the weight function $\varrho$, i.e.

$$
\varrho>0
$$

of the metric $\mathbf{g}$ as a bilinear form for space tangent vectors $\xi$, i.e.

$$
\xi \neq 0 \Rightarrow \xi^{\mu} g_{\mu \nu} \xi^{\nu}>0,
$$

and of the matrix $\phi$ (and hence also its inverse $\phi^{-1}$ ) as a sesquilinear form for an $m$-dimensional space of vectors $u$ with components $u^{a}$, i.e.

$$
u \neq 0 \Rightarrow \bar{u}^{\dot{a}} \phi_{\dot{a} b} u^{b}>0 .
$$

The required inequality (2.13) results directly from the postulates (2.14), (2.15), (2.16) by the general principle that a tensor product of positive definite bilinear or sesquilinear forms (in the present instance, the tensor with components $\left.\varrho^{-1} g_{\mu \nu} \phi_{[1] a b} \phi_{[0]}^{-1 c \dot{d}}\right)$ is always itself a positive definite bilinear or sesquilinear form 
for the tensor product of the corresponding vector spaces (in the present instance, the space containing the current matrix with components $J_{v c}^{b}$ and conjugate components $\left.J^{*}{ }_{\mu \dot{i}}{ }^{a}\right)$.

It thus follows from Green's theorem that if the boundary conditions are such that the relevant surface integral tends to zero there, i.e.

$$
\oint_{S} \varrho \Delta^{; \mu} d S_{\mu} \rightarrow 0
$$

(where $d S_{\mu}$ denotes the components of the metric normal surface element) then we must have

$$
\stackrel{\odot}{\mathbf{J}}^{\mu}=0,
$$

and hence, by (2.9),

$$
\Delta=\mathbf{C},
$$

where $\mathbf{C}$ is a constant matrix, throughout the base space region under consideration. To obtain an absolute uniqueness theorem of form

$$
\Delta=0
$$

it is then sufficient that there should exist at least one part of the boundary on which the limiting value of $\phi$ is specified uniquely so that $\Delta$ is zero there. It is to be noted that the requirement (2.17) does not mean that the gradient $\Delta^{; \mu}$ itself must tend to zero on the boundary: an alternative possibility is that the weight function $\varrho$ should have a vanishing limit.

\section{General Consistency Requirements for the Mazur Construction}

Despite the apparent simplicity of the requirements (2.1), (2.2), and (2.4) on which our short ("royal road") derivation of the Mazur identity in the form (2.11) was based, the range of circumstances to which it can be applied is not quite so large as might first appear, since consistency requirements for the system (2.4) will impose severe restrictions if the linearly independent components of $\phi$ are not free field variables but subject to non-linear restraints, as they must be if $\phi$ is to satisfy the positivity requirement (2.16). In order to obtain a self consistent system in which this requirement is satisfied, let us work in terms of a variational formulation, based on a simple Lagrangian scalar function

$$
L=\frac{1}{2} \varrho^{-1} g_{\mu \nu} \operatorname{tr}\left\{\mathbf{J}^{* \mu} \cdot \boldsymbol{\phi} \cdot \mathbf{J}^{\nu} \cdot \boldsymbol{\phi}^{-1}\right\}
$$

with the current as given by (2.1), which leads directly to field equations of the form (2.4) in the free (unconstrained) case. This Lagrangian is manifestly real by (2.2), and furthermore it will have a positivity property analogous to (2.13), namely

$$
\mathbf{J}^{\mu} \neq 0 \Rightarrow L>0,
$$

when the same positivity postulates as before, namely (2.14), (2.15), (2.16) are satisfied. 
Starting from the more compact (though no longer manifestly real and positive) equivalent form

$$
L=\frac{1}{2} \varrho^{-1} \operatorname{tr}\left\{\mathbf{J}^{\mu} \cdot \mathbf{J}_{\mu}\right\},
$$

one immediately obtains the effect of varying the field $\phi$ in the form

$$
\delta L=\varrho^{-1} \operatorname{tr}\left\{\mathbf{J}^{\mu} \cdot \delta \mathbf{J}_{\mu}\right\}=\operatorname{tr}\left\{\mathbf{J}^{\mu} \cdot \boldsymbol{\phi}^{-1} \cdot \delta \boldsymbol{\phi}\right\}_{; \mu}-\operatorname{tr}\left\{\mathbf{J}_{; \mu}^{\mu} \cdot \boldsymbol{\phi}^{-1} \cdot \delta \boldsymbol{\phi}\right\} .
$$

The variational principle therefore takes the form of the requirements that one should hàve

$$
\operatorname{tr}\left\{\mathbf{J}_{; \mu}^{\mu} \cdot \boldsymbol{\phi}^{-1} \cdot \delta \boldsymbol{\phi}\right\}=0
$$

for arbitrary allowed $\delta \phi$, which would immediately lead to field equations of the required form (2.4) if there were no constraint.

If the matrix $\phi$ is constrained to lie on some lower dimensional variety within the vector space of all hermitean matrices, the variational condition (3.5) will not lead to such a strong set of field equations as (2.4), but an obvious way to recover the full set would be to require that variations of $\phi$ should be generated by (let us say) the right action of some subgroup $\mathscr{G}$ of $\mathrm{GL}(m)$ matrices, since in this case $\phi^{-1} \cdot \delta \phi$ as well as the current matrices $\mathbf{J}^{\mu}$ and their divergence $\mathbf{J}^{\mu}{ }_{; \mu}$ would all belong to the algebra $\mathscr{A}$ of $\mathscr{G}$. Thus (3.5) would boil down to the requirement that the contraction of $\mathbf{J}_{; \mu}^{\mu}$ with any other arbitrarily chosen element of the algebra should be zero, which provided the latter were semi-simple, would lead again to the required result (2.4).

The situation with which we are concerned cannot be quite as straighforward as that described in the previous paragraph since a simple right (or left) matrix group action will not in general preserve the hermicity property (2.2). This property will however be preserved if we suppose that the subspace to which $\phi$ is constrained is generated by an action of the form

$$
\boldsymbol{\phi} \mapsto \mathbf{Q}^{*} \cdot \boldsymbol{\phi} \cdot \mathbf{Q},
$$

where $\mathbf{Q}$, with components $\mathbf{Q}_{b}^{\alpha}$, is any element in the matrix group $\mathscr{G}$ under consideration. The corresponding infinitesimal variations will therefore have the form

$$
\delta \phi=\left(\phi \cdot \mathbf{a}+\mathbf{a}^{*} \cdot \boldsymbol{\phi}\right) \delta \lambda,
$$

where $\delta \lambda$ is an arbitrary infinitesimal parameter variation and $\mathbf{a}$, with components $a_{b}^{a}$, is any element of the algebra $\mathscr{A}$ of generators of $\mathscr{G}$.

It is convenient at this stage to introduce a notation scheme based on the involutory ( $\phi$ dependent) automorphism

$$
\mathbf{b} \mapsto \mathbf{b}^{\dagger}
$$

of the space of $m \times m$ matrices $\mathbf{b}$ with mixed components $b^{a}{ }_{b}$ according to the specification

$$
\mathbf{b}^{\dagger}=-\boldsymbol{\phi}^{-1} \cdot \mathbf{b}^{*} \cdot \boldsymbol{\phi}
$$

where the minus sign is included so as to ensure that its effect on a commutator is given straightforwardly by

$$
[\mathbf{b}, \mathbf{c}]^{\dagger}=\left[\mathbf{b}^{\dagger}, \mathbf{c}^{\dagger}\right]
$$


Since the hermiticity property (2.2) implies the reciprocity property

$$
\left(\mathbf{b}^{\dagger}\right)^{\dagger}=\mathbf{b}
$$

that characterises an involution, any such (mixed) $m \times m$ matrix b can be unambiguously decomposed in the form

$$
\mathbf{b}=\mathbf{b}^{+}+\mathbf{b}^{-},
$$

where the odd and even parts are characterised by

$$
\left(\mathbf{b}^{ \pm}\right)^{\dagger}= \pm \mathbf{b}^{ \pm}
$$

and hence are given explicitly by

$$
\mathbf{b}^{ \pm}=\frac{1}{2}\left(\mathbf{b} \pm \mathbf{b}^{\dagger}\right) .
$$

The commutators between the odd and even subspaces constructed in this way will have the properties

$$
\left[\mathbf{b}^{+}, \mathbf{c}^{+}\right]^{-}=\left[\mathbf{b}^{-}, \mathbf{c}^{-}\right]^{-}=\left[\mathbf{b}^{+}, \mathbf{c}^{-}\right]^{+}=0 .
$$

In terms of the standard (pseudo) metric

$$
\langle\mathbf{b}, \mathbf{c}\rangle=\frac{1}{2} \operatorname{tr}\{\mathbf{b} \cdot \mathbf{c}\}
$$

on the whole vector space of mixed $m \times m$ matrices, the elements of the even and odd subspaces have norms given respectively by

$$
\left\langle\mathbf{b}^{ \pm}, \mathbf{b}^{ \pm}\right\rangle=\mp \frac{1}{2} \operatorname{tr}\left\{\mathbf{b}^{ \pm} \cdot \boldsymbol{\phi}^{-1} \cdot \mathbf{b}^{ \pm *} \cdot \boldsymbol{\phi}\right\},
$$

so that [by the same principle as was used in deriving (2.13) and (3.2)] one can deduce, as a direct consequence of the positivity property (2.16) postulated for $\phi$, that the metric is respectively negative and positive definite on the even and odd subspaces, i.e.

and

$$
\mathbf{b}^{+} \neq 0 \Rightarrow\left\langle\mathbf{b}^{+}, \mathbf{b}^{+}\right\rangle<0,
$$

$$
\left.\mathbf{b}^{-} \neq 0 \Rightarrow\left\langle\mathbf{b}^{-}, \mathbf{b}^{-}\right\rangle\right\rangle 0 \text {. }
$$

In terms of this scheme, the identity (2.3) satisfied by the current matrix is expressible in the equivalent compact forms

$$
\mathbf{J}_{\mu}^{\dagger}=-\mathbf{J}_{\mu}, \quad \mathbf{J}_{\mu}^{+}=0,
$$

from which it follows that its derivative must satisfy

$$
\left.\left(\mathbf{J}_{\mu ; \nu}\right)^{+}=\frac{1}{2} \varrho^{-1}\left[\mathbf{J}_{\mu}, \mathbf{J}_{v}\right]=\varrho\left(\varrho^{-1} \mathbf{J}_{[\mu}\right) ; v\right],
$$

where the latter equality expresses the integrability condition for the existence of a matrix $\phi$ such that the current takes the form (2.1). The manifestly antisymmetric right-hand side contributions in (3.21) will drop out when it is contracted, so that it may be seen that the current divergence has the same oddness property as the current itself, which is expressible by the equivalent relations

$$
\left(\mathbf{J}_{\mu}^{; \mu}\right)^{+}=0, \quad \mathbf{J}_{\mu}^{; \mu}=\left(\mathbf{J}_{\mu}{ }^{; \mu}\right)^{-} .
$$


Since the postulated form (3.7) of the allowed variations is expressible in the present terminology as the requirement that we should have

$$
\boldsymbol{\phi}^{-1} \cdot \delta \phi=2 \mathbf{a}^{-} \delta \lambda, \quad \mathbf{a} \in \mathscr{A},
$$

the variational condition (3.5) reduces to the form

$$
\left\langle\left(\mathbf{J}_{\mu} ; \mu\right)^{-}, \mathbf{a}^{-}\right\rangle=0, \quad \forall \mathbf{a} \in \mathscr{A} .
$$

Now since we know from (3.19) that the metrics induced on the even and odd subspaces $\mathscr{A}^{ \pm}$, as defined by

$$
\mathscr{A}^{ \pm}=\left\{\mathbf{a}^{ \pm}: \mathbf{a} \in \mathscr{A}\right\}
$$

are respectively negative and positive definite and hence non-degenerate in either case, we can conclude from (3.24) and (3.22) that

$$
\mathbf{J}_{; \mu \mu}^{\mu} \neq 0 \Rightarrow\left(\mathbf{J}_{; \mu}^{\mu}\right)^{-} \notin \mathscr{A}^{-} .
$$

However this last eventuality is not excluded by any of the assumptions we have made so far: although the restricted form (3.23) of the allowed variations implies by (2.2) that the current itself should have the form

$$
\mathbf{J}_{\mu}=\mathbf{a}_{\mu}^{-}
$$

for some one form $\mathbf{a}_{\mu}$ with Lie algebra valued components, i.e.

$$
\mathbf{a}_{\mu} \subset \mathscr{A},
$$

it does not automatically follow that the current $\mathbf{J}_{\mu}$ itself, or for that matter the subspace $\mathscr{A}^{-}$, should lie within $\mathscr{A}$.

In order to make progress we are therefore obliged at this stage to introduce what is in fact a severe further restriction by postulating that our system be such that wherever a belongs to the algebra $\mathscr{A}$ of $\mathscr{G}$, the same should necessarily hold for $\mathbf{a}^{\dagger}$, i.e.

$$
\mathbf{a} \in \mathscr{A} \Rightarrow \mathbf{a}^{\dagger} \in \mathscr{A},
$$

so that the same applies to the even and odd parts, i.e.

$$
\mathscr{A}^{-} C \mathscr{A}, \mathscr{A}^{+} C \mathscr{A} \text {. }
$$

Under these circumstances (3.27) and (3.28) imply

$$
\mathbf{J}_{\mu} \subset \mathscr{A},
$$

and hence also

$$
\mathbf{J}_{\mu}{ }^{; \mu} \subset \mathscr{A},
$$

since differentiation automatically preserves the property of belonging to the (fixed) algebra $\mathscr{A}$ (though not of belonging to the $\phi$ dependent subspaces $\mathscr{A}^{ \pm}$). Thus the eventuality (3.26) can at last be excluded, so that one finally obtains the desired conservation law (2.1). 


\section{Realisation of the Requirements for the Mazur Construction}

In order to fulfill the requirements described in the preceding section we now confine our attention to matrix groups $\mathscr{G}$ of the standard kind, as defined by the requirement that they should leave invariant some standard tensor $\boldsymbol{\eta}$ with components of the form $\eta^{a b}$, which at this stage need not be supposed to satisfy any particular symmetry or antisymmetry conditions. This means that a matrix $\mathbf{Q}$ in $\mathscr{G}$, with components of the form $Q^{a}{ }_{b}$ will be characterised by

$$
\mathbf{Q} \cdot \boldsymbol{\eta} \cdot \mathbf{Q}^{*}=\boldsymbol{\eta} .
$$

Subject to the proviso that $\boldsymbol{\eta}$ be non-singular, it can be used for index raising and lowering in accordance with the conventions described e.g. by the author [16], where the ordering must be treated carefully except in the real-symmetric case. Thus for any $m$-dimensional vector $u$ or covector $v$ with conjugate components $u_{\dot{a}}, v^{\dot{a}}$ respectively one defines

$$
u^{a}=\eta^{a b} u_{b}, \quad v_{a}=v^{b} \eta_{b a},
$$

where the lowered component version of $\boldsymbol{\eta}$ is defined as the inverse of its hermitian conjugate, i.e. by

$$
\eta_{\dot{a}}^{b}=\eta_{\dot{a} c} \eta^{* c b}=\delta_{\dot{a}}^{b}
$$

or equivalently by

$$
\eta^{* a}{ }_{b}=\eta^{* a c} \eta_{c b}=\delta_{b}^{a}
$$

This convention enables us to write the defining property (4.1) of the group $\mathscr{G}$ in component notation as

$$
Q_{\dot{a} c} Q^{* c b}=\delta_{\dot{a}}^{b}
$$

The corresponding defining property for the elements a with components of the form $a_{b}^{a}$, of the algebra $\mathscr{A}$ of $\mathscr{G}$ is expressible as

$$
\mathbf{a} \cdot \boldsymbol{\eta}+\boldsymbol{\eta} \cdot \mathbf{a}^{*}=0
$$

or equivalently, in component notation

$$
a_{i c} \eta_{b}^{c}+a_{i b}^{*}=0 \text {. }
$$

This property obviously implies that one has

$$
a_{b}^{a} a_{a}^{b}=a_{i}^{*}{ }^{b} a_{b}^{*}{ }^{\dot{a}},
$$

and hence by the definition (3.16) that the metric on the algebra has the reality property

$$
\langle\mathbf{a}, \mathbf{b}\rangle=\overline{\langle\mathbf{a}, \mathbf{b}\rangle} .
$$

For any element characterised by (4.6) the automorphism (3.9) is expressible by

$$
\mathbf{a}^{\dagger}=(\boldsymbol{\eta} \cdot \phi)^{-1} \cdot \mathbf{a} \cdot(\boldsymbol{\eta} \cdot \boldsymbol{\phi})
$$

where $\boldsymbol{\eta} \cdot \boldsymbol{\phi}$ is simply the matrix with components $\phi^{a}{ }_{b}$. The crucial postulate (3.29) to the effect that the defining property (4.6) of the algebra should be preserved by 
an automorphism of this form for allowed values of $\phi$ can be seen to be equivalent to the requirement that we should have the commutation property

$$
\left[(\boldsymbol{\eta} \cdot \boldsymbol{\phi}) \cdot\left(\boldsymbol{\eta} \cdot \boldsymbol{\phi}^{*}\right), \mathbf{a}\right]=0
$$

for any element a of the algebra.

It can be seen from (4.10) that the scalar product [as defined by (3.16)] of any elements $\mathbf{a}, \mathbf{b}$ of the algebra $\mathscr{A}$ will satisfy

$$
\langle\mathbf{a}, \mathbf{b}\rangle=\left\langle\mathbf{a}^{\dagger}, \mathbf{b}^{\dagger}\right\rangle,
$$

and hence that the cross product between the odd and even algebra subspaces [as characterised by (3.24)] will be zero, i.e. one will have the orthogonality property

$$
\left\langle\mathbf{a}^{+}, \mathbf{b}^{-}\right\rangle=0 \text {. }
$$

In order to satisfy (4.11) for an arbitrary element $\mathbf{a} \in \mathscr{A}$, we are now lead to impose the requirement that the allowed values of $\phi$ should satisfy

$$
(\boldsymbol{\eta} \cdot \boldsymbol{\phi}) \cdot\left(\boldsymbol{\eta} \cdot \boldsymbol{\phi}^{*}\right)=\kappa \mathbf{1}
$$

for some scalar constant $\kappa$. It can be checked that this condition is automatically preserved (with the value of $\kappa$ remaining invariant) by the group action (3.6) for any $\mathbf{Q}$ satisfying the group characterisation (4.1). The postulated condition (4.14) can be expressed in component notation as

$$
\phi_{\dot{a} c} \phi^{* c b}=\kappa \eta^{*}{ }_{a}^{b} .
$$

Comparing this with the analogous component version (4.5) of the group characterisation condition, we see that it will be identical, i.e. the postulate (4.14) will be equivalent to

$$
\boldsymbol{\eta} \cdot \boldsymbol{\phi} \in \mathscr{G},
$$

if (and only if) the standard tensor $\boldsymbol{\eta}$ is subject to a condition of the form

$$
\boldsymbol{\eta}=\kappa \boldsymbol{\eta}^{*},
$$

which entails the requirement

and implies

$$
|\kappa|^{2}=1
$$

$$
\eta^{a}{ }_{b}=\kappa \delta_{b}^{a}, \quad \eta^{*}{ }_{i}^{b}=\bar{\kappa} \delta_{\dot{a}}^{b} .
$$

In the case of a real matrix group, the requirement (4.18) can be satisfied only for

$$
\kappa= \pm 1
$$

the two possibilities corresponding respectively to (pseudo) orthogonal and symplectic groups. In the case of complex matrix groups it is possible, without affecting the group, to adjust a phase factor in the specification of $\boldsymbol{\eta}$, and hence of $\kappa$, so as to obtain the standard hermiticity property as specified by $\kappa=+1$.

From this stage on we shall suppose that the group $\mathscr{G}$ under consideration is indeed of (pseudo) hermitian, (pseudo) orthogonal or symplectic type, with $\boldsymbol{\eta}$ satisfying (4.17) and (4.20). There is then no further loss of generality in supposing 
that the constant $\kappa$ in (4.14) is the same as that specified by (4.17) because it can be seen [by taking the trace of (4.14) and using the positivity property (2.16)] that it could deviate by at most a constant positive factor which can be normalised to unity by a choice of scale. Despite the apparent generality of the requirements (2.1) and (2.16) their realisation has lead us to postulate a rather special situation in which $\phi_{a b}$ is restricted not only by the positivity condition (2.16) but by the more particular requirement that the allowed values of the corresponding mixed tensor

$$
\phi_{b}^{a}=\eta^{a \dot{c}} \phi_{\dot{c} b}
$$

should lie on an orbit of the adjoint action

$$
\boldsymbol{\eta} \cdot \boldsymbol{\phi} \mapsto \mathbf{Q}^{-1} \cdot(\boldsymbol{\eta} \cdot \boldsymbol{\phi}) \cdot \mathbf{Q}
$$

that expresses the effect of (3.6) for a group $\mathscr{G}$ of the kind characterised by (4.1).

The space of allowed values of $\phi$, as constructed in this way, will automatically have a natural Riemannian metric induced by the algebra metric (3.16) according to the specification

$$
\begin{aligned}
d s^{2} & =\frac{1}{4}\left\langle(\boldsymbol{\eta} \cdot \boldsymbol{\phi})^{-1} \cdot d(\boldsymbol{\eta} \cdot \boldsymbol{\phi}),(\boldsymbol{\eta} \cdot \boldsymbol{\phi})^{-1} \cdot d(\boldsymbol{\eta} \cdot \boldsymbol{\phi})\right\rangle \\
& =\frac{1}{4}\left\langle\boldsymbol{\phi}^{-1} \cdot d \boldsymbol{\phi}, \boldsymbol{\phi}^{-1} \cdot d \boldsymbol{\phi}\right\rangle .
\end{aligned}
$$

Since the allowed variations have the form (3.23), the positivity property

$$
\left.d \phi \neq 0 \Rightarrow d s^{2}=\left\langle\mathbf{a}^{-}, \mathbf{a}^{-}\right\rangle \delta \lambda^{2}\right\rangle 0
$$

holds as an application of (3.19). The induced metric (4.23) is evidently invariant under the adjoint action (4.22) of the group on the orbit. More particularly it will be invariant under the action

$$
\mathbf{Q} \mapsto \mathbf{Q}^{\dagger}
$$

of the group on itself that is induced by an arbitrarily chosen fixed element $\boldsymbol{\eta} \cdot \boldsymbol{\phi}$ on the orbit, according to the specification

$$
\mathbf{Q}^{\dagger}=(\boldsymbol{\eta} \cdot \boldsymbol{\phi})^{-1} \cdot \mathbf{Q} \cdot(\boldsymbol{\eta} \cdot \boldsymbol{\phi}) .
$$

Any element a of the algebra will determine a field of infinitesimal allowed displacements

$$
\mathbf{Q} \mapsto \mathbf{Q}+d \mathbf{Q}
$$

within the orbit, which by (3.7) and (4.6) will have the form

$$
d \mathbf{Q}=[\mathbf{Q}, \mathbf{a}] \delta \lambda
$$

for any fixed value of $d \lambda$. This isometry generating (i.e. Killing) vector field will by transformed by (4.26) onto another such field of displacements

$$
\mathbf{Q}^{\dagger} \mapsto \mathbf{Q}^{\dagger}+d \mathbf{Q}^{\dagger},
$$

which will have the form

$$
d \mathbf{Q}^{\dagger}=\left[\mathbf{Q}^{\dagger}, \mathbf{a}^{\dagger}\right] \delta \lambda
$$

where, in conformity with the notation scheme previously introduced, the corresponding transformed value $\mathbf{a}^{\dagger}$ of a will be precisely as specified by (4.10). The 
isometry (4.26) evidently leaves the chosen fixed element $\boldsymbol{\eta} \cdot \boldsymbol{\phi}$ itself invariant and therefore determines an isomorphism of its tangent space. This isomorphism can be seen to map a displacement

$$
d(\boldsymbol{\eta} \cdot \boldsymbol{\phi})=[\boldsymbol{\eta} \cdot \boldsymbol{\phi}, \mathbf{a}] d \lambda
$$

in the tangent space onto an element which, by (4.10) will be given by

$$
d(\boldsymbol{\eta} \cdot \boldsymbol{\phi})^{\dagger}=\left[\boldsymbol{\eta} \cdot \boldsymbol{\phi}, \mathbf{a}^{\dagger}\right] d \lambda=[\boldsymbol{\eta} \cdot \boldsymbol{\phi},-\mathbf{a}] d \lambda=-d(\boldsymbol{\eta} \cdot \boldsymbol{\phi}),
$$

i.e. this isometry reverses all the tangent vectors at the arbitrarily chosen fixed point $\boldsymbol{\eta} \cdot \boldsymbol{\phi}$ on the allowed group orbit. This establishes the well known fact [17] that such group orbits are totally symmetric spaces in the technical sense that there exists a tangent space inverting isometry about every point.

\section{The Lorentzian Signature Case}

The totally symmetric orbit spaces that have just been set up can be interpreted as quotient spaces of the form $\mathscr{G} / \mathscr{H}$, where $\mathscr{H}$ is the isotropy subgroup of $\mathscr{G}$ that leaves some arbitrarily chosen element $\boldsymbol{\eta} \cdot \boldsymbol{\phi}$ of the orbit invariant. In view of the fact that the action of $\mathscr{G}$ has the form (3.6) where $\phi$ is positive definite, the elements of the isotropy subgroup must also belong to the (strictly) unitary or (strictly) orthogonal group of all matrices that leave such a positive definite form invariant in the complex and real cases respectively, so that we shall have respectively

or

$$
\begin{aligned}
\mathscr{H} & =\mathscr{G} \cap U(m), \\
\mathscr{H} & =\mathscr{G} \cap O(m) .
\end{aligned}
$$

In the case $\kappa=+1$, for a hermitian or symmetric metric $\eta$ with signature such that its diagonal form contains $p$ negative and $q$ positive components (with $p+q=m$ ) we shall have the respective possibilities

$$
\mathscr{G} / \mathscr{H}=\mathrm{SU}(p, q) / \mathrm{S}(\mathrm{U}(p) \times \mathrm{U}(q))
$$

or

$$
\mathscr{G} / \mathscr{H}=\mathrm{SO}(p, q) / \mathrm{S}(\mathrm{O}(p) \times \mathrm{O}(q)),
$$

while in the real symplectic case, $\kappa=-1$, (which needs an even value of $m$ ) we shall have

$$
\mathscr{G} / \mathscr{H}=\mathrm{Sp}(m) / \mathrm{OSp}(m) .
$$

The examples considered explicitly by Mazur $[3,17]$ were of the former type (5.3) with Lorentzian signature as characterised by $\kappa=+1$. In such a case, matrices of the form

$$
\phi_{\dot{a} b}=\eta_{\dot{a} b}+2 \bar{v}_{\dot{a}} v_{b}
$$

will have the required positivity property (2.16) for any sufficiently large timelike vector $v$, or to be more precise, whenever

$$
\bar{v}^{a} v_{a}<-\frac{1}{2} \text {. }
$$


More particularly, a matrix of the form (5.6) will satisfy the required group membership requirement (4.15), which in this case reduces simply to,

$$
\phi_{a c} \phi^{c b}=\delta_{a}^{b}
$$

whenever $v$ is a timelike unit vector, i.e.

$$
\bar{v}^{a} v_{a}=-1,
$$

which is consistent with the positivity requirement (5.7). Since this property is preserved by the relevant group action, the conditions (5.6) and (5.9) characterise all elements of the allowed orbit.

For any two distinct such fields, $\phi_{[0]}$ and $\phi_{[1]}$, the corresponding value of $\Delta$, which in this case is defined by

$$
\delta_{\dot{b}}^{\dot{a}}+\Delta_{\dot{a}}{ }^{b}=\phi_{[1] \dot{a} c} \phi_{[0]}^{c b}
$$

will be given in terms of the corresponding vector $v$ which is defined only to within a phase factor) by

$$
\frac{1}{2} \Delta_{\dot{a}}^{b}=\bar{v}_{[1] \dot{a}} v_{[1]}^{b}+2 v_{[1] c} \bar{v}_{[0]}^{c} \bar{v}_{[1] \dot{a}} v_{[0]}^{b}+v_{[0] \dot{a}} v_{[0]}^{b},
$$

and hence its trace is given by

$$
\frac{1}{4} \Delta=\left|v_{[1] c} \bar{v}_{[0]}\right|^{2}-1,
$$

which satisfies the positivity condition

$$
\phi_{[1]} \neq \phi_{[0]} \Rightarrow \Delta>0 .
$$

The metric (4.23) on such a space will take the form

$$
d s^{2}=\frac{1}{8} \phi^{a b}\left(d \phi_{b \dot{b}}\right) \phi^{c \dot{d}} d \phi_{d a}=\gamma_{a b} d v^{\dot{a}} d \vec{v}^{b},
$$

where we have introduced the notation

$$
\gamma_{a b}=\eta_{a b}+\bar{v}_{\dot{a}} v_{b}
$$

for the effective metric whose restriction to the allowed hyperboloid characterised by (5.9) is the same as the metric induced on it directly by $\eta_{i b}$. The distance $s$ along a geodesic within such a hypersurface can be seen to be given by

$$
\cosh s=\left|v_{[1] c} \bar{v}_{[0]}^{c}\right|,
$$

so that it will be related to the corresponding value of $\Delta$, characterising the separation between the endpoints, by

$$
\Delta=4 \sinh ^{2} s .
$$

Since the matrix $\phi$ obtained from (5.6) is not affected by an overall phase change in $v$, and since its magnitude is in any case fixed by (5.9) it follows that only $m-1$ of its $m$ complex components correspond to the $(2 m-2)$ real degrees of freedom of the field. Their relation to the variables of the standard formulation [5] of the black hole equilibrium problem for the pure vacuum $(m=2)$ and electromagnetic $(m=3)$ cases is specified in Appendix B. 


\section{The Bunting Identity}

Having elucidated the way in which the very elegant Mazur construction is dependent on a particularly high degree of symmetry in the space of variables, we now come to describe a more robust and general procedure that has been developed independently for the same purpose by Bunting.

The basic Bunting construction as described in this section does not require that the space of variables should have any special symmetry properties at all, but merely that the field equations are a homogeneous quadratic function of the space gradients of the form of harmonic type in the sense of being obtainable from a variational principle based on a Lagrangian scalar that is locally a homogeneous quadratic function of the gradients of the independent field variables $X^{A}$ $(A=1, \ldots, N$, say) of the form

$$
L=\frac{1}{2} \varrho g^{\mu v} \hat{g}_{A B} X^{A}{ }_{, \mu} X^{B}{ }_{, v},
$$

where $g^{\mu v}$ is as usual the inverse of the $n$-dimensional base space metric field with components $g_{\mu \nu}$ in terms of the base space coordinates $x^{\mu}$, and $\varrho$ is a scalar field on the base space which could be absorbed into the metric by a conformal rescaling except in the special case $n=2$ with which the black hole application is in fact concerned. The coefficients $\hat{g}_{A B}$ are required to be independent of position on the base space, though they may have arbitrary dependence on the field variables $X^{A}$, and they may therefore be interpreted as components of a metric of the form

$$
d s^{2}=\hat{g}_{A B} d X^{A} d X^{B}
$$

on an $N$ dimensional manifold $\mathscr{X}$ of which the $X^{A}$ are local coordinates. In its most general sense a harmonic system may be envisaged as a mapping

$$
\mathscr{M} \rightarrow \mathscr{X}
$$

of the $n$-dimensional base Riemannian (or pseudo-Riemannian) manifold $\mathscr{M}$ onto an $N$-dimensional Riemann (or pseudo-Riemannian) manifold $\mathscr{X}$ whose local coordinate representation

$$
x^{\mu} \mapsto X^{A}
$$

satisfies the equations derived from the Lagrangian that is given by (5.1) in terms of the corresponding local coordinate components of the metrics on $\mathscr{M}$ and $\mathscr{X}$. A very helpful introduction (from a physicist's point of view) to the theory of such mappings has been provided by Misner [18].

In any discussion of maps (whether of harmonic or more general type, such as those discussed in [30]) between spaces $\mathscr{M}$ and $\mathscr{X}$ that are both endowed with metrics, or even merely with linear connections, it is convenient to introduce the corresponding concept of covariant differentiation over $\mathscr{M}$ of elements specified in the tangent space (or its tensor products) at the image points $X(x) \in \mathscr{X}$ of base space points $x \in \mathscr{M}$. The covariant variation $\delta \vec{V}$, say of a tangent space element $\vec{V}$, say between nearby points with position coordinates $X^{A}$ and $X^{A}+d X^{A}$ on $\mathscr{X}$, is given in terms of the variations $d V^{A}$ of the corresponding components $V^{A}$ of $\vec{V}$ by an expression of the usual form

$$
(\delta \vec{V})^{A}=d V^{A}+\left(d X^{C}\right) \hat{\Gamma}_{C}{ }_{B} V^{B},
$$


where $\hat{\Gamma}_{C}{ }_{B}$ are the components of the linear connection on $\mathscr{X}$. In the Riemannian (or pseudo-Riemannian) case these components will in turn be given in terms of the coordinate components $\hat{g}_{A B}$ and $\hat{g}^{A B}$ of the metric on $\mathscr{X}$ by an expression of the familiar form

$$
\hat{\Gamma}_{C}^{A}{ }_{B}=\hat{g}^{A D}\left(g_{D(C, B)}-\frac{1}{2} g_{C B, D}\right),
$$

where a comma denotes partial differentiation. The case of covariant differentiation over the base space $\mathscr{M}$ arises when the variation $d X^{A}$ is considered as representing the variation of the image $X(x)$ arising from a corresponding infinitesimal displacement between nearby points with coordinates $x^{\mu}$ and $x^{\mu}+d x^{\mu}$ in the base space, i.e. when

$$
d X^{A}=X^{A}{ }_{, \mu} d x^{\mu} .
$$

The corresponding expression for the covariant derivative of $\vec{V}$ can thus be seen to have the form

$$
\nabla_{\mu} V^{A}=V_{, \mu}^{A}+\Gamma_{\mu}^{A}{ }_{B} V^{B},
$$

where the components of the (mapping dependent) connection form $\Gamma_{\mu}$ over $\mathscr{M}$ are given by

$$
\Gamma_{\mu}^{A}{ }_{B}=X^{C}{ }_{, \mu} \hat{\Gamma}_{C}{ }_{B}^{A} .
$$

The formula can be applied to cases where $\vec{V}$ is defined only at the image points of the mapping (6.4), but in the particular case when $\vec{V}$ is specified in advance as a vector field over $\mathscr{X}$, the formula (5.8) can be expressed in terms of the ordinary covariant derivative

$$
\hat{\nabla}_{B} V^{A}=V^{A}{ }_{B}+\hat{\Gamma}_{B}{ }^{A}{ }_{C} V^{C}
$$

over $\mathscr{X}$ by the simple relation

$$
\nabla_{\mu} V^{A}=X_{, \mu}^{C} \hat{\nabla}_{C} V^{A} .
$$

The commutator of two covariant differentiation operation of the form (6.8) takes the form

$$
\left[\nabla_{\mu}, \nabla_{v}\right] V^{A}=R_{\mu \nu B}^{A} V^{B},
$$

and the components of the (mapping dependent) curvature two-form $\mathbf{R}_{\mu \nu}$ over $\mathscr{M}$ that is defined in this way will be given by

$$
R_{\mu \nu}{ }_{B}^{A}=X^{C}{ }_{, \mu} X^{D}{ }_{, \nu} \hat{R}_{C D}{ }_{B}^{A},
$$

where the ordinary Rieman tensor on $\mathscr{X}$ is defined by the usual relation

$$
\left[\hat{\nabla}_{C}, \hat{\nabla}_{D}\right] V^{A}=\hat{R}_{C D}{ }_{B}{ }_{B} V^{B} \text {. }
$$

The foregoing concept of covariant differentiation of tangent vectors on the image space $\mathscr{X}$ can naturally be extended in the normal way to the associated cotangent vectors and tensors on $\mathscr{X}$. In conjunction with the ordinary concept of covariant differentiation on the base space, which we shall denote simply by a semi-colon, so that for example

$$
v_{; v}^{\mu}=v^{\mu}{ }_{, v}+\Gamma_{v}^{\mu} \varrho_{\varrho},
$$


where

$$
\Gamma_{v \varrho}^{\mu}=g^{\mu \lambda}\left(g_{\lambda(v, \varrho)}-\frac{1}{2} g_{v \varrho, \lambda}\right),
$$

the generalised covariant differentiation procedure can be further extended in a natural manner to bi-tensors defined on tensor products of tangent or cotangent spaces on $\mathscr{X}$ and $\mathscr{M}$. Thus in the particular case of the projection bitensor, with components

$$
\nabla_{\mu} X^{A}=X_{, \mu}^{A},
$$

which behaves simultaneously as a vector on $\mathscr{X}$ and a covector on $\mathscr{M}$, we shall have

$$
\nabla_{\mu} \nabla_{v} X^{A}=X_{, v, \mu}^{A}-\Gamma_{\mu}{ }^{e}{ }_{v} X^{A}{ }_{, \varrho}+\Gamma_{\mu B}^{A} X^{B}{ }_{, v}=\left(\nabla_{v} X^{A}\right)_{; \mu}+\hat{\Gamma}_{B}{ }^{A}{ }_{c}\left(\nabla_{\mu} X^{B}\right) \nabla_{v} X^{C} .
$$

On applying this formalism to the particular case of a harmonic mapping, as specified by the Lagrangian (6.1), which may now be expressed more compactly as

$$
L=\frac{1}{2} \varrho\left(\nabla_{\mu} X^{A}\right) \nabla^{\mu} X_{A},
$$

we obtain

$$
\delta L=\left\{\varrho\left(\nabla^{\mu} X_{A}\right) \delta X^{A}\right\}_{; \mu}-\left\{\nabla_{\mu}\left(\varrho \nabla^{\mu} X_{A}\right)\right\} \delta X^{A} .
$$

Thus one obtains the harmonic field equations in the compact form

$$
\nabla_{\mu}\left(\varrho \nabla^{\mu} X^{A}\right)=0
$$

or more explicitly

$$
\left(\varrho X^{A}{ }_{, \mu}\right)^{; \mu}=-\varrho X^{B}{ }_{, \mu} X^{C ; \mu} \hat{\Gamma}_{B}{ }^{A}{ }_{C} .
$$

Although such a system of equations has come to be commonly designated by the description "harmonic" it would perhaps be preferable to use the more explicit and less overworked adjective "hypergeodesic" in view of the fact that it is a natural higher dimensional generalisation of the ordinary geodesic equations, which are included as the special case for which the base space $\mathscr{M}$ is uni-dimensional, and also to avoid confusion with the non-covariant system of equations

$$
\left(X_{, \mu}^{A}\right)^{; \mu}=0
$$

that characterises what are widely known as "harmonic" coordinates. This latter system will however be compatible with the hypergeodesic Eq. (6.22) in the case in which $\mathscr{X}$ has the same dimension as $\mathscr{M}$ and in which, instead of being specified in advance, the metric on $\mathscr{X}$ is directly induced by the mapping, according to the prescription

$$
\hat{g}^{A B}=g^{\mu \nu} X_{, \mu}^{A} X_{\nu}^{A}
$$

In this isometric case, and for a uniform value of the weight factor $\varrho$, the hypergeodesic Eq. (6.22) reduce to

$$
\left(X_{, \mu}^{A}\right)^{; \mu}=\|\hat{g}\|^{-1 / 2}\left(\|\hat{g}\|^{1 / 2} g^{A B}\right)_{, B},
$$

which will be satisfied automatically (since the right-hand side is interpretable as the Laplacian of the new coordinates $X^{A}$ in terms of themselves), expressing the fact that an isometry is a trivial special case of a hypergeodesic mapping. The further (non-covariant) requirement that the right-hand side of (6.25) should 
vanish [leaving (6.23) as a consequence] is De Donder's well known intrinsic characterisation of a "harmonic" coordinate system.

Returning from this digression, we are now ready to describe the basic Bunting construction which applies to the case in which one has two distinct but homotopic fields $X_{[0]}(x), X_{[1]}(x)$ corresponding to a pair of mappings $\mathscr{M} \rightarrow \mathscr{X}$ given as

$$
x^{\mu} \mapsto X_{[0]}^{A}, \quad x^{\mu} \mapsto X_{[1]}^{A}
$$

say, between spaces $\mathscr{M}$ and $\mathscr{X}$ having fixed values for the respective metrices $g_{\mu \nu}$ and $\hat{g}_{A B}$. The essential idea is to work with a suitable homotopy $X(x ; t)$ between the two fields corresponding to a mapping $\mathscr{M} \times I \mapsto \mathscr{X}$ given as

$$
\left(x^{\mu} ; t\right) \mapsto X^{A},
$$

where $t$ is a parameter in the unit interval $I$, ranging from 0 to 1 , representing the affine length along a curve $I \rightarrow \mathscr{X}$ that is chosen, for each base point $x \in \mathscr{M}$, to have initial and final endpoints at the corresponding image points, so that we have

$$
X^{A}(x ; 0)=X_{[0]}^{A}(x), \quad X^{A}(x ; 1)=X_{[1]}^{A}(x) .
$$

At a later stage we shall need to impose the further requirement that each such curve $I \rightarrow \mathscr{X}$ be geodesic, i.e. that its total length

$$
s=\int_{0}^{1}\left(s_{A} s^{A}\right)^{1 / 2} d t
$$

should be stationary under small endpoint preserving variations, where we have introduced the notation

$$
s^{A}=\frac{d X^{A}}{d t}
$$

for the affine tangent vector along the curve as normalised with respect to the affine parameter $t$. For the time being however, we only need to require that the curve varies smoothly as a function of the base point $x \in \mathscr{M}$, thereby determining some correspondingly smooth scalar length field over $\mathscr{M}$. Since the length interval $d s$ along each curve must evidently be related to the corresponding affine parameter variation by the proportionality relation

$$
d s=s d t,
$$

the tangent vector $\vec{s}(x ; t)$ must satisfy the $t$ independent normalisation condition

$$
s_{A} s^{A}=s^{2} .
$$

It is convenient at this stage to introduce a dot notation to indicate covariant differentiation with respect to $t$ along each curve, so that for a vector $\vec{V}$, for example, we shall have

$$
\dot{V}^{A}=\frac{d V^{A}}{d t}+s^{B} \Gamma_{B}^{A}{ }_{C} V^{C}
$$

In doing this we deviate slightly from the original treatment of Bunting $[1,2]$, in which a dot was used for the straight (i.e. non-covariant) operation $d / d t$ of 
derivation with respect to $t$ at fixed base space position $x \in \mathscr{M}$. For the case of the scalar position element $X(x ; t)$, which simply gives

$$
\dot{X}^{A}=s^{A},
$$

the distinction does not arise, but it is important for the second derivative,

$$
\ddot{X}^{A}=\dot{S}^{A},
$$

which will be required to vanish later on when we impose the geodicity condition.

Applying the covariant differentiation procedure described at the beginning of this section to the mapping $\mathscr{M} \rightarrow \mathscr{X}$ that is specified by (6.27) for any fixed value of $t$, one can verify that the dot derivation operation commutes with the covariant base space gradient operation when applied to the effectively scalar coordinates $X^{A}$, i.e. one has

$$
\nabla_{\mu} s^{A}=\left(\nabla_{\mu} X^{A}\right)^{\cdot} .
$$

However when these operations are successively applied to a vector they no longer commute unless $\mathscr{X}$ is flat: in general one obtains

$$
\nabla_{\mu} \dot{V}^{A}-\left(\nabla_{\mu} V^{A}\right)^{\cdot}=X^{C}{ }_{, \mu} s^{D} \hat{R}_{C D}{ }_{B}{ }_{B} V^{D}
$$

where, in accordance with (6.12), the image space curvature components are given by

$$
\hat{R}_{C D}{ }_{B}^{A}=2 \hat{\Gamma}_{[D|B,| C]}^{A}+2 \hat{\Gamma}_{\left[C{ }^{A}{ }_{E} \hat{\Gamma}_{\mid D]}{ }^{E}{ }_{B}\right.} .
$$

Applying this in conjunction with (6.36) one obtains the useful commutation identity

$$
\nabla_{\mu}\left(\varrho \nabla_{v} s^{A}\right)-\left(\nabla_{\mu}\left(\varrho \nabla_{v} X^{A}\right)\right)^{\cdot}=-\varrho \hat{R}_{B C D}^{A} S^{C}\left(\nabla_{v} X^{B}\right) \nabla_{\mu} X^{D} .
$$

The foregoing machinery can now be used to give a very rapid derivation of the Bunting identity, which is based [1,2] on an evaluation of the (density weighted) base space Laplacian of the field $s^{2}$ (which plays a role analogous to that of the deviation matrix trace $\Delta$ in the Mazur identity). To start with we shall have

$$
s s_{, \mu}=\frac{1}{2} \nabla_{\mu} s^{2}=s_{A} \nabla_{\mu} s^{A}
$$

by (6.32) (where the right-hand side may be evaluated at any value of $t$ ), and hence

$$
\left(\varrho s s_{, \mu}\right)^{; \mu}=\varrho\left(\nabla^{\mu} s_{A}\right) \nabla_{\mu} s^{A}+s^{A} \nabla^{\mu}\left(\varrho \nabla_{\mu} s^{A}\right) .
$$

The last term here can be processed using (6.39) so as to give

$$
\begin{aligned}
\left(\varrho S S_{, \mu}\right)^{; \mu}= & \left(s^{A} \nabla_{\mu}\left(\varrho \nabla_{v} X^{A}\right)\right)^{\cdot}-\dot{s}^{A} \nabla_{\mu}\left(\varrho \nabla^{\mu} X^{A}\right) \\
& +\varrho\left\{\left(\nabla^{\mu} s_{A}\right) \nabla_{\mu} s^{A}-\hat{R}_{A B C D} s^{A} S^{C}\left(\nabla_{\mu} X^{B}\right) \nabla_{v} X^{D}\right\} .
\end{aligned}
$$

Integrating over the curve for a fixed base space element $x \in \mathscr{M}$ and using the fact that the left-hand side (unlike the separate contribution on the right) is constant along the curve, one obtains the identity

$$
\begin{gathered}
\left(\varrho S s_{, \mu}\right)^{; \mu}-\left[s_{A} \nabla_{\mu}\left(\varrho \nabla^{\mu} X^{A}\right)\right]_{0}^{1}+\int_{0}^{1} d t \dot{s}_{A} \nabla_{\mu}\left(\varrho \nabla^{\mu} X^{A}\right) \\
=\varrho \int_{0}^{1} d t\left\{\left(\nabla^{\mu} s_{A}\right) \nabla_{\mu} s^{A}-\hat{R}_{A B C D} S^{A}\left(\nabla_{\mu} X^{B}\right) s^{C} \nabla^{\mu} X^{D}\right\}
\end{gathered}
$$


which holds for any smooth (affine) homotopy between mappings, regardless of any field equations that may be satisfied. If one now chooses the homotopy curves so as to satisfy the geodesic equations

$$
\dot{s}^{A}=0,
$$

assuming that such a choice is possible, then the application of the harmonic (or, more strictly, hypergeodesic) field equations (6.21) at the endpoints, which gives

$$
\left[s_{A} \nabla_{\mu}\left(\varrho \nabla^{\mu} X^{A}\right)\right]_{0}^{1}=0
$$

can be seen to reduce the left-hand side of (6.43) to a divergence of the required form. Then provided the image space has a positive difinite metric, i.e.

$$
\vec{V} \neq 0 \Rightarrow \hat{g}_{A B} V^{A} V^{B}>0,
$$

and non-positive curvature, i.e.

$$
\hat{R}_{A B C D} W^{A C} W^{B D} \leqq 0
$$

for any $W^{A B}$, then it will follow from our previous postulates (2.14) and (2.15) of positivity of the base space metric with components $g_{\mu \nu}$ and of the scalar density $\varrho$, that the right-hand side of (6.43) can never be negative, and that in order for it to vanish one must have $\nabla_{\mu} s^{A}$ vanishing everywhere as well, which by (6.40) is only possible if the gradient of $s$ itself vanishes. Thus one obtains a conclusion of the desired form

$$
s_{, \mu} \neq 0 \Rightarrow\left(\varrho s s_{, \mu}\right)^{; \mu}>0 .
$$

Hence if the boundary conditions are such that

$$
\oint_{S} \varrho s s^{; \mu} d S_{\mu} \rightarrow 0,
$$

one can conclude that the metric separation $s$ between the two solutions is uniform,

$$
s=c,
$$

where $c$ is a constant. More particularly we can conclude that this constant vanishes, i.e. that

$$
s=0
$$

everywhere, if there is at least one part of the boundary at which the value of the field is specified completely, which means that the solution will be unique, at least within the relevant geodesic homotopy class.

\section{The Applicability of the Bunting Construction and its Relationship to the Special Mazur Case}

In the particular case of a image manifold $\mathscr{X}$ with positive definite metric and nonpositive curvature [in accordance with (6.46) and (6.47)] as required for obtaining the conclusions (6.48), (6.51) of the previous section, the existence of the postulated geodesic homotopy is not such a severe restriction as it would be otherwise: Bunting has shown that it in order for such a homotopy to exist (and be unique) it is 
sufficient (as a consequence of the well known theorem [17] that the exponential map is an isomorphism in such a case) for the space $\mathscr{X}$ to be simply connected and geodesically complete. [For metrics whose signature is indefinite there can exist point pairs unconnected by any geodesic even in the geodesically complete case, while for metrics with locally positive Riemannian curvature there can exist several distinct geodesics connecting a given pair of points even in the simply connected case. The well known uniqueness of the connecting geodesic in the positive metric but negative curvature case can be regarded as the application of the foregoing conclusion (6.51) for 1-dimensional base spaces].

Furthermore, when the base space $\mathscr{M}$ is itself simply connected, then the additional requirement of simple connectedness of the image space $\mathscr{X}$ becomes superfluous, since $\mathscr{X}$ will in any case have a simply connected universal covering space containing a geodesic homotopy between the corresponding lifted mappings, so that a (not necessarily unique) local geodesic homotopy can always be obtained by projection. Provided $\mathscr{M}$ is simply connected, any such local geodesic homotopy can be extended in an unambiguous manner over the whole of $\mathscr{M}$.

The requirement that the allowed range of variables constituting the image space $\mathscr{X}$ should be geodesically complete can also be relaxed: it is evidently sufficient that this allowed range should be extendable in such a way as to become part of a larger space having the required properties of completeness, non-positive curvature, etc. For a strictly homogeneous Riemannian space, completeness holds automatically.

Although the class of non-linear $\sigma$-models with totally symmetric image spaces to which the Mazur construction can be applied (as described in Sect. 4) is evidently a very specialised subset of the full class of harmonic (i.e. hypergeodesic) mappings onto image spaces $\mathscr{X}$ which in general need give no particular symmetry properties at all, one can nevertheless raise the question of whether the Mazur identity might be able to settle some uniqueness questions beyond the scope of the Bunting identity in view of the fact that the application of the latter depends on the non-positivity requirement (6.47) on the curvature, whereas there was no need for any explicit mention of the curvature at all in the application of the Mazur identity. In fact, however, the answer to this question is negative: the Mazur construction cannot be used to evade the need to invoke the inequality (6.47) because of the fact [17] that the non-negative curvature property is actually built in automatically for symmetric spaces having the particular kind of structure (as the quotient space of a non-compact group $\mathscr{G}$ by a maximal compact subgroup $\mathscr{H}$ ) described in Sect. 4.

To see this explicitly, we start from the remark that each element a in the Lie algebra $\mathscr{A}$ of generators of a Lie group $\mathscr{G}$ of isometries (if there are any) of a Riemannian (or pseudo-Riemannian) space $\mathscr{X}$ will be associated with a corresponding Killing vector field $\vec{a}$, with components $a^{A}$, which will satisfy, the Killing equations

$$
\hat{\nabla}_{(A} a_{B)}=0
$$

(which express the requirement that the Lie derivative with respect to $\vec{a}$ of the metric $\hat{g}$ on $\mathscr{X}$ should vanish) and hence also the integrability condition

$$
\hat{\nabla}_{C}\left(\hat{V}_{B} a_{A}\right)=\hat{R}_{A B C D} a^{D} \text {. }
$$


Using the fact that the displacement commutator field $[\overrightarrow{a, b}]$ associated with $[\mathbf{a}, \mathbf{b}]$ in $\mathscr{A}$ is given simply by reversing the ordinary Lie differentiation commutator $[\vec{a}, \vec{b}]$ (the latter meaning the Lie derivative of $\vec{b}$ with respect to $\vec{a}$ ), so that one has

$$
[a, b]^{A}=b^{B} \hat{V}_{B} a^{A}-a^{B} \hat{V}_{B} b^{A},
$$

one can deduce that for any three such generators $\mathbf{a}, \mathbf{b}, \mathbf{c}$ we shall have

$$
[[a, b], c]^{A}=\hat{R}_{B C D}^{A} c^{B} b^{C} a^{D}+2 c^{D}\left(\hat{V}_{C} a^{[A}\right) \hat{V}_{D} b^{C]}-[a, b]^{B} \hat{V}_{B} c^{A} .
$$

Let us now restrict our attention to the case in which $\mathscr{X}$ is a totally symmetric space in the technical sense that there is an isometric involution centred on each point $X \in \mathscr{X}$ that reverses the tangent space at that point. For any particular point, $X_{[0]}$ say, the corresponding involution maps any Killing vector field $\vec{a}$ onto another Killing vector field $\vec{a}^{\dagger}$ say, thereby inducing a corresponding involution on the isometry group Lie algebra $\mathscr{A}$ (of the kind exemplified by (4.10) in the particular application with which we shall be concerned). The resulting decomposition properties, of the form expressed already by our Eqs. (3.10)-(3.15), will reflect corresponding decomposition properties for the Killing vector fields themselves. Thus (with respect to an arbitrarily chosen point) any Killing vector field $\vec{a}$ can be decomposed into odd and even parts $\vec{a}^{-}$and $\vec{a}^{+}$, which will themselves be Killing fields, given [in analogy with (3.13)] by the specification

$$
a^{ \pm A}=\frac{1}{2}\left(a^{A} \pm a^{\dagger A}\right)
$$

and the odd and even Killing vector fields will have commutation properties of the form (3.15), i.e.

$$
\left[a^{+}, b^{+}\right]^{-A}=\left[a^{-}, b^{-}\right]^{-A}=\left[a^{+}, b^{-}\right]^{+A}=0,
$$

the first of these, expressing the closure of the even subset, being an obvious reflection of the fact that the even Killing vectors generate the isotropy subgroup associated with the fixed point $X_{[0]}$ of the involution. In view of the fact that the tangent space at $X_{[0]}$ is reversed by involution, the even Killing vector fields must evidently vanish at that particular point, i.e.

$$
a_{[0]}^{+A}=0,
$$

and it can be seen (taking account of the fact that the metric is invariant under the inversion) that their covariant derivatives of even order must also vanish there. The odd Killing vector fields associated with the point $X_{[0]}$ will not themselves vanish there, but it can be seen that their covariant derivatives of odd order must do so, so that in particular, in the first order case, we obtain

$$
\hat{V}_{B} a^{-A}{ }_{[0]}=0 \text {. }
$$

Hence [by substituting odd fields on the left-hand side of (7.4) and using (7.8) and (7.7) to simplify the result] we see that the curvature of a symmetric space can be expressed directly in terms of commutators in the form

$$
\left(\hat{R}_{B C D}^{A} a^{B} b^{C} c^{D}\right)_{[0]}=\left[\left[a^{-}, b^{-}\right], c^{-}\right]_{[0]}^{A} .
$$


This specifies the curvature completely in view of the fact that the tangent space will be completely spanned by Killing vectors in any homogeneous space, and hence in particular, in any totally symmetric space.

We now restrict ourselves further to situations (such as that considered in Sect. 4) in which the metric is induced directly by the standard metric on the group algebra according to a formula of the form

$$
\left(\hat{g}_{A B} a^{A} b^{B}\right)_{[0]}=\left\langle\mathbf{a}^{-}, \mathbf{b}^{-}\right\rangle=\alpha \operatorname{tr}\left\{\mathbf{a}^{-} \cdot \mathbf{b}^{-}\right\},
$$

where $\alpha$ a proportionality constant. It then follows from (7.9) [and from the commutation rules (3.15) corresponding to (7.6)] that the curvature will be given by

$$
\left(\hat{R}_{A B C D} a^{A} b^{B} c^{C} d^{D}\right)_{[0]}=\alpha \operatorname{tr}\left\{\left[\left[\mathbf{a}^{-}, \mathbf{b}^{-}\right], \mathbf{c}^{-}\right], \mathbf{d}^{-}\right\}=\alpha \operatorname{tr}\left\{\left[\mathbf{a}^{-}, \mathbf{b}^{-}\right]^{+},\left[\mathbf{c}^{-}, \mathbf{d}^{-}\right]^{+}\right\},
$$

and hence in particular

$$
\left(\hat{R}_{A B C D} a^{A} b^{B} a^{C} b^{D}\right)_{[0]}=\alpha\left\langle\left[\mathbf{a}^{-}, \mathbf{b}^{-}\right]^{+},\left[\mathbf{a}^{-}, \mathbf{b}^{-}\right]^{+}\right\rangle .
$$

In the case of a compact group [such as the $\mathrm{SO}(3)$ isometry group of the ordinary 2-sphere] for which the trace of products on the algebra is negative definite, the proportionality factor $\alpha$ would have to be chosen negative in order to give a positive definite metric $\hat{g}$, and hence as a result the curvature would be nonnegative also. However the conditions of applicability of the Mazur construction require that we should be dealing with a situation in which the trace of products is negative definite only on the even subalgebra subspace, while being positive definite on the odd algebra subspace, as an expression of the fact that the symmetric spaces under considerations are quotients of a non-compact group by a maximal compact subgroup. In such a situation one needs a positive choice of the proportionality factor $\alpha$ (our normalisation convention took $\alpha=\frac{1}{2}$ ) so as to obtain a group metric that is positive definite [in accordance with (3.19)] on the odd algebra subspace $\mathscr{A}^{-}$, in order to give rise [by (7.10)] to a positive definite metric on the symmetric quotient space $\mathscr{X}$. This restricts the group metric to be negative definite [in accordance with (3.18)] on the even subalgebra $\mathscr{A}^{+}$, so that it then follows from (7.12), which expresses the curvature in terms of a product of even subalgebra elements (obtained as commutators of members of the odd subspace $\mathscr{A}^{-}$) that it must automatically be non-positive in accordance with Bunting's requirement (6.47).

This completes the demonstration that the negative curvature property used by Bunting's method of establishing uniqueness is in fact an automatic consequence of the totally symmetric character of these particular non-linear $\sigma$-models. It is evident however that the negative curvature requirement (which would be preserved by sufficiently small but nevertheless finite perturbations) does not in itself entail such a strong restriction as total symmetry. One therefore sees that the latter is not really essential for the uniqueness properties, contrary to the impression that might have been obtained from the Mazur derivation [3], and contrary to what is in fact the case for other properties such as the infinite sequence of conservation laws discussed, e.g. by Eichenherr and Forger [20] and de Vega [21] for which the total symmetry really is essential. Symmetry is also an essential requirement for the construction of gauge-coupled non-linear $\sigma$-models [22] for 
which one can obtain a natural generalisation of the Mazur construction in a manner to be described elsewhere [30].

\section{Appendix A. The Black Hole Equilibrium Boundary Value Problem}

Subject to a number of reasonably plausible physical assumptions (including technical restrictions which rule out degenerate limit cases such as the MajumdarPapapetrou black-holes of Hartle and Hawking [15]) and using a considerable body of previous mathematical work $[6-8,11,12,23-26]$ the present author was able to reduce the 4-dimensional geometric problem of finding the equilibrium states of a black hole equilibrium solution of the Einstein-Maxwell (electromagnetic vacuum) solutions in an asymptotically flat background to an elliptic boundary value problem four unknowns $X, Y, E, B$, (two in the non-electromagnetic case) on a two-dimensional base space with a known metric

$$
d s_{\mathrm{II}}^{2}=\frac{d \lambda^{2}}{\lambda^{2}-c^{2}}+\frac{d \mu^{2}}{1-\mu^{2}} .
$$

The base space covers the ellipsoidal coordinate range

$$
-1<\mu<1, \quad c<\lambda<\infty
$$

and the boundary conditions are specified in terms of the scale parameter $c$ which has the dimensions of mass, and a second parameter $J$ representing the angular momentum, a third parameter $Q$ representing the total electric charge and, if one so desires, a fourth parameter $P$ representing the (mathematically if not physically conceivable) total magnetic monopole moment. (In so far as the space time geometry is concerned this fourth parameter is redundant, since the metric is invariant under the duality transformations which preserve the sum of squares $P^{2}+Q^{2}$.) The necessary boundary conditions at large radius, $\lambda \rightarrow \infty$, are given by

$$
\begin{gathered}
E=Q \mu+O\left(\lambda^{-1}\right), \quad B=-P \mu+O\left(\lambda^{-1}\right), \\
Y=2 J \mu\left(3-\mu^{2}\right)+O\left(\lambda^{-1}\right), \\
X=\left(1-\mu^{2}\right) \lambda^{2}+O(\lambda) .
\end{gathered}
$$

The boundary conditions on the horizon, $\lambda \rightarrow c$, consist merely of the requirement that the unknown functions, $E, B, Y, X$ should be regular functions of $\lambda$ and $\mu$ there. The most delicate boundary conditions are those on the north and south polar axes of symmetry $\mu \rightarrow 1$ and $\mu \rightarrow-1$, where it is required not only that the unknown $E, B, Y, X$, should be regular as functions of $\lambda$ and $\mu$ but more specifically that they should satisfy

$$
\begin{gathered}
\frac{\partial E}{\partial \lambda}=O\left(1-\mu^{2}\right), \quad \frac{\partial B}{\partial \lambda}=O\left(1-\mu^{2}\right) \\
\frac{\partial Y}{\partial \lambda}=O\left(1-\mu^{2}\right), \quad \frac{\partial Y}{\partial \mu}+2\left(E \frac{\partial B}{\partial \mu}-B \frac{\partial E}{\partial \mu}\right)=O\left(1-\mu^{2}\right) \\
X=O\left(1-\mu^{2}\right) \frac{\left(\mu^{2}-1\right)}{2} \frac{1}{X} \frac{\partial X}{\partial \mu}=1+O\left(1-\mu^{2}\right)
\end{gathered}
$$


This last equation is merely an expression of the requirement that although $X$ itself must vanish on the symmetry axis $\mu^{2}=1$, its derivative with respect to $\mu$ must not. [This condition was printed incorrectly - by omission of the factor $\frac{1}{2}\left(\mu^{2}-1\right)$-in the final version of the boundary conditions in the original derivation [4], and some confusion may have arisen from transcription of that erroneous version in other work [9], where the derivation was not discussed].

The field equations are of the Ernst [26] form, which was shown [4] to be obtainable from a Lagrangian scalar of the harmonic type (6.19) where the base space scalar field $\varrho$ is given by

$$
\varrho^{2}=\left(\lambda^{2}-c^{2}\right)\left(1-\mu^{2}\right),
$$

and where the image space metric is given by

$$
d s^{2}=\frac{(d X)^{2}+(d Y+2 E d B-2 B d E)^{2}}{4 X^{2}}+\frac{(d E)^{2}+(d B)^{2}}{X} .
$$

Whereas the potentials $Y, E, B$, are defined in an indirect manner ( $E$ and $B$ being zero in the non-electromagnetic case, while $Y$ and $B$ are zero in the strictly static case) the variable $X$ is directly interpretable geometrically as the squared length of the primary space time Killing vector on which the Ernst [26] formulation of the Einstein-Maxwell equations is based. The standard version of the Ernst formulation uses as its starting point the generator of the stationary symmetry, which is timelike, with negative squared length at large asymptotic distances, which vanishes on an ergosurface (where the corresponding Ernst equations would become singular) within which (except in the strictly case) it becomes timelike. The present formulation [4] was designed to avoid the difficulty of the ergosurface singularity by using as a starting point the generator of the axisymmetry. This axisymmetry Killing vector is guaranteed to be non-vanishing throughout the domain (A.2) under consideration by the physical requirement of causality (no closed timelike curves) which ensure that it is strictly spacelike,

$$
X>0 \text {, }
$$

except on the axis itself where of course it vanishes in accordance with (A.8). As a result the metric (A.13) is strictly positive definite, with signature $(++++)$ [whereas the standard formulation would have given a metric with indefinite signature $(++--)$ in the outer region where the stationary Killing vector is timelike]. Although this non-standard formulation saves us from having to cope with an ergosurface singularity in the interior of the domain (A.2) that is of interest, it has not eliminated it altogether: we instead have to deal with a singularity on the axis $\mu^{2} \rightarrow 1$ where the denominators in (A.10) tend to zero. (As an additional price we also have to use the rather unfamiliar and awkward form (A.3)-(A.5) of asymptotic boundary conditions, but as an unexpected bonus the horizon boundary conditions become as simple as could be imagined). However although the singularity makes the treatment of the axis rather delicate, it is rendered tractable by the fact that the asymptotic boundary conditions (A.3), (A.4) enable us to deduce that, like $X$ (which vanishes), the other variables $Y, E, B$, are also fully determined along the entire length of the symmetry axis: by integrating along the 
axis using (A.6) and (A.7), one obtains

$$
\begin{gathered}
Y=4 J \mu+O\left(1-\mu^{2}\right), \\
E=Q \mu+O\left(1-\mu^{2}\right), \\
B=-P \mu+O\left(1-\mu^{2}\right),
\end{gathered}
$$

as $\mu^{2} \rightarrow 1$. Hence for two distinct solutions the difference must vanish on the axis, i.e. using the notation convention introduced in Sect. (2),

$$
\begin{aligned}
X^{\odot} & =O\left(1-\mu^{2}\right), & Y^{\odot} & =O\left(1-\mu^{2}\right), \\
E^{\odot} & =O\left(1-\mu^{2}\right), & B^{\odot} & =O\left(1-\mu^{2}\right) .
\end{aligned}
$$

\section{Appendix B. Translation from the Ernst Formulation to the Mazur Formulation}

Mazur's reformulation [18] of the problem posed in Appendix A was based on Kinnersley's transformation [27] of the Ernst variables into a system which could be interpreted as a standard (3-complex dimensional) linear representation of the symmetric group SU(2, 1). Starting from standard complex Ernst [26] variables

$$
\begin{gathered}
\psi=E+i B, \\
\varepsilon=-X+i Y-|\psi|^{2} .
\end{gathered}
$$

Kinnersley introduced a new set of three complex variables $v_{0}, v_{1}, v_{2}$ given up to an arbitrary overall complex factor by

$$
\frac{v_{1}+v_{0}}{v_{1}-v_{0}}=\varepsilon, \quad \frac{v_{2}}{v_{1}-v_{0}}=\psi
$$

In terms of a 3 dimensional Lorentz signature metric $\eta_{a b}$ of the standard Minkowski form, $\operatorname{diag}(-1,1,1)$, a vector $v_{a}$ with components specified (up to an arbitrary complex factor) by (B.3) can be seen to have squared length given by

$$
v_{c} \bar{v}^{c} \equiv \eta_{\dot{a} b} v^{\dot{a}} \bar{v}^{b}=-X\left|v_{1}-v_{0}\right|^{2},
$$

and the image space metric (A.10) will be expressible as

$$
d s^{2}=\frac{|d \varepsilon+2 \bar{\psi} d \psi|^{2}}{4 X^{2}}+\frac{|d \psi|^{2}}{X}=\frac{\left|v_{a} d \bar{v}^{a}\right|^{2}}{\left(v_{c} \bar{v}^{c}\right)^{2}}-\frac{d v_{a} d \bar{v}^{a}}{v_{c} \bar{v}^{c}},
$$

where the latter form is manifestly invariant under the $\operatorname{SU}(2,1)$ transformations that preserve the Minkowski form of the metric. In the pure vacuum case, for which $\psi$ is zero, this formulation reduces to the standard 2-complex dimensional representation of the $\mathrm{SU}(1,1)(\equiv \mathrm{SO}(2,1))$ symmetry group discovered by Geroch [28] and recently discussed in a wider context by Sanchez [29]. The expression (B.5) simplifies if we restrict the indeterminacy of $v^{a}$ by imposing a normalisation condition of the form.

$$
v_{c} \bar{v}^{c}=\mp 1 \text {, }
$$

where the upper, i.e. negative, sign choice can be seen from (B.4) to be necessary when the inequality (A.14) holds (as a result of using a non-standard Ernst 
formulation based on a spacelike space-time Killing vector) whereas the lower, i.e. positive, sign would be necessary if one were using the standard Ernst formulation based on a timelike space-time Killing vector for which one would have $X<0$. Subject to (B.6), the final expression in (B.5) reduces to

$$
d s^{2}=\left( \pm \eta_{\dot{a} b}+\bar{v}_{\dot{a}} v_{b}\right) d v^{\dot{a}} d \bar{v}^{b}
$$

which agrees with (5.14) for the upper choice sign, resulting from (A.14). For either choice of sign we can construct a hermitian matrix

$$
\phi^{a b}=\eta^{a b} \mp 2 \bar{v}^{a} v^{b},
$$

which will satisfy

$$
\phi^{a b} \phi_{b c}=\delta_{c}^{a},
$$

and give

$$
d s^{2}=\frac{1}{8} \operatorname{tr}\left\{(\phi \cdot d \phi)^{2}\right\}
$$

in accordance with (5.8) and (5.14), but this matrix will only satisfy the positivity requirement (2.16) for the upper choice of sign resulting from (A.14), for which (B.8) will agree with (5.6).

It can be seen that if the Kinnersley transformation (B.3) were applied to the metric (A.13) in the case of a standard timelike Killing vector based Ernst formulation with $X<0$ we would obtain a symmetric space of the form $\mathrm{SU}(2,1) / \mathrm{S}(\mathrm{U}(1,1) \times \mathrm{U}(1))$ instead of the form $\mathrm{SU}(2,1) / \mathrm{S}(\mathrm{U}(2) \times \mathrm{U}(1))$, and although there would still be a Mazur identity of the form (2.11), it would no longer satisfy the positivity condition (2.13). [This would not necessarily prevent the solution from being unique: indeed in the strictly static case the more obvious formulation leading to a harmonic mapping into a space with an (non-compact) $\mathrm{SU}(1,1)$ isotropy subgroup is ultimately equivalent to our present formulation in terms of a harmonic mapping onto a space with a (maximally compact) SU(2) isotropy subgroup.]

A convenient choice of phase gives an explicit expression for $v_{a}$, subject to the normalisation condition (B.6) as

$$
\left(v_{0}, v_{1}, v_{2}\right)=\frac{1}{2}|X|^{-1 / 2}(\varepsilon-1, \varepsilon+1,2 \psi) .
$$

The product of the vectors $v_{[0]}{ }^{a}$ and $v_{[1]}{ }^{a}$ arising from distinct solutions will have the form

$$
v_{[1] c} \bar{v}_{[0]}{ }^{c}=\frac{-\frac{1}{2} \tilde{X}-\frac{1}{2}\left|\psi^{\odot}\right|^{2}+i\left(\frac{1}{2} Y^{\odot}+B_{[1]} E_{[0]}-B_{[0]} E_{[1]}\right)}{\langle X\rangle},
$$

where (in loose accord with our previous notation convention [5])

$$
\langle X\rangle=\left(X_{[0]} X_{[1]}\right)^{1 / 2}, \quad \tilde{X}=X_{[0]}+X_{[1]},
$$

and we have

$$
\left|\psi^{\odot}\right|^{2}=\left(E^{\odot}\right)^{2}+\left(B^{\odot}\right)^{2} .
$$

Hence by (5.11) we obtain the trace of the deviation matrix in the form

$$
\Delta=\frac{\left(X^{\odot}\right)^{2}+2 \tilde{X}\left|\psi^{\odot}\right|^{2}+\left|\psi^{\odot}\right|^{4}+\left(Y^{\odot}+\tilde{E} B^{\odot}-\tilde{B} E^{\odot}\right)^{2}}{\langle X\rangle} .
$$


As a consequence of the boundary conditions (A.3)-(A.5) in the previous section, it will satisfy

$$
\Delta=O\left(\lambda^{-2}\right), \quad \frac{\partial \Delta}{\partial \lambda}=O\left(\lambda^{-3}\right)
$$

as $\lambda \rightarrow \infty$, and as a consequence of (A.12) to (A.16) it will be regular on the axis,

$$
\Delta=O(1), \quad \frac{\partial \Delta}{\partial \mu}=O(1)
$$

as $\mu^{2} \rightarrow 1$ despite the vanishing of the denominator in (B.15). Regularity on the horizon,

$$
\Delta=O(1), \quad \frac{\partial \Delta}{\partial \lambda}=O(1)
$$

as $\lambda \rightarrow c$, obviously follows automatically from the requirement that the individual variables $E, B, Y, X$, are regular since the latter remains non-zero there. It therefore follows from the form of (A.1) and (A.2) that the Mazur requirement (2.17), or alternatively [taking account of (5.17)] the Bunting requirement (6.49), will be satisfied, and hence that the solution to the problem posed in Appendix A is unique.

Acknowledgements. The author wishes to thank Prof. E. D. Fackerell and Dr. G. W. Gibbons for helpful discussions concerning the Bunting and Mazur methods respectively, and also to thank Dr. G. L. Bunting for having made available the unpublished draft manuscript of his contribution to Sect. 12 of the $2^{\text {nd }}$ Australasian Mathematics Convention on the $14^{\text {th }}$ May 1981.

\section{References}

1 Bunting, G.: The black hole uniqueness theorem, in (unpublished) Proc. of the $2^{\text {nd }}$ Australasian Mathematics Convention Sydney, 11-15 May 1981

2. Bunting, G.: Proof of the uniqueness conjecture for black holes. PH. D. Thesis, Department of Mathematics, University of New England. Armidale, N.S.W. 1983

3. Mazur, P.O.: Proof of uniqueness of the Kerr-Newman black hole solution. J. Phys. A 15, 3173 (1982)

4. Carter, B.: In: black holds. De Witt, B., De Witt, C., eds. New York: Gordon and Breach 1973

5. Carter, B.: In: general relativity. Hawking, S.W., Israel, W., eds Cambridge: Cambridge University Press 1979

6. Carter, B.: Axisymmetric black hole has only two degrees of freedom. Phys. Rev. Lett. 26, 331 (1971)

7. Hawking, S.W.: Black holes in the Brans-Dicke theory of gravitation. Commun. Math. Phys. $25,152(1972)$

8. Hawking, S.W., Ellis, G.F.R.: The large scale structure of space-time. Cambridge: Cambridge University Press 1973

9. Robinson, D.C.: Uniqueness of the Kerr black hole. Phys. Rev. Lett. 34, 905 (1975)

10. Robinson, D.C : Classification of black holes with electromagnetic fields. Phys. Rev. D 10, 458 (1974)

11. Israel, W.: Event horizons in static vacuum space-times. Phys. Rev. 164, 1776 (1967)

12. Israel, W.: Event horizons in static electrovac space-times. Commun. Math. Phys. 8, 245 (1968)

13. Müller zum Hagen, H., Robinson, D.C., Seifert, H.J.: Black holes in static vacuum space-times. Gen. Rel. Grav. 4, 53 (1973)

14. Robinson, D.C.: A simple proof of the generalization of Israel's theorem. Gen. Rel. Grav. 8, 695 (1977) 
15. Hartle, J.B., Hawking, S.W : Solutions of the Einstein-Maxwell equations with many black holes. Commun. Math. Phys. 26, 87 (1972)

16. Carter, B.: In: recent developments in gravitation (Cargèse, 1978) Nato advanced study institutes series B44. Lévy, M., Deser, S., eds. New York: Plenum 1979

17. Kobyashi, S., Nomizu, K.: Foundations of differential geometry, Vol. 11, New York: Interscience 1969

18 Mazur, P.O.: A relationship between the electrovacuum Ernst equations and nonlinear $\sigma$-model. Acta Phys. Pol B 14, 219 (1983)

19 Misner, C.W.: Harmonic maps as models for physical theories. Phys. Rev. D 18, 4510 (1978)

20. de Vega, H.J.: Field theories with an infinite number of conservation laws and Bäcklund transformations in two dimensions. Phys. Lett. B 87, 233 (1979)

21. Eichenherr, H., Forger, $M$ : More about non-linear sigma models on symmetric spaces. Nucl. Phys. B 164, 528 (1980)

22. Madore, J.: Gauge-invariant extension of the $\sigma$-model with electromagnetic interactions. Phys. Rev. D 15, 514 (1977)

23. Lichnerowicz, A.: Theories relativistes de la gravitation et de l'electromagnétisme, Paris: Masson 1955

24. Papapetrou, A.: Champs gravitationnels stationnaires à symétrie axiale. Ann. Inst. H. Poincaré A 4, 83 (1966)

25. Carter, B.: Killing horizons and orthogonal transitive groups in space-time. J. Math. Phys. 10, 70 (1969)

26. Ernst, F.J.: New formulation of the axially symmetric gravitational field problem. II Phys. Rev. 168, 1415 (1968)

27. Kinnersley, W : Generation of stationary Einstein-Maxwell fields J. Math Phys. 14, 651 (1973)

28. Geroch, R.: A method for generating solutions of Einstein's equations. J. Math. Phys. 12, 918 (1971)

29. Sanchez, N.: Connection between the nonlinear $\sigma$-model and the Einstein equations of general relativity. Phys. Rev. D 26, 2589 (1982)

30. Carter, B : Gauge coupling of non-linear $\sigma$-model and a generalised Mazur identity. In: Non-linear equation in classical and quantum field theory. Vol 223, p. 72. Sanchez, N, ed. Lecture Notes in Physics Berlin, Heidelberg, New York: Springer 1985

Communicated by R Stora

Received February 24, 1984; in revised form December 19, 1984 
